\title{
Begleitung will gelernt sein - Qualifizierungskonzept für die Rolle der methodischen Begleitung
}

\author{
Joana Jungclaus, Petra A. Arndt und Agnes Bauer
}

Die agile Lernform agiles Sprintlernen wurde im Rahmen des BMBF-geförderten Vorhabens inMEDIASres im Zeitraum von 2017 bis 2020 von den Forschungsinstituten Fraunhofer IAO und ZNL TransferZentrum für Neurowissenschaften und Lernen entwickelt und gemeinsam mit vier Unternehmen zu jeweils mehreren Lernthemen erprobt.

Die Rolle der methodischen Begleitung wurde vorwiegend intern von den Unternehmen besetzt. Begleitend zu den Praxiserprobungen haben die Entwicklerinnen des agilen Sprintlernens dafür insgesamt elf Personen aus den vier Partnerunternehmen zum methodischen Begleiter ausgebildet. Sie wurden im Rahmen der Umsetzungen durch das Forscherteam gecoacht und begleitet.

Dieses Kapitel beschreibt das Vorgehen für die Qualifizierung der methodischen Begleiter, wie es im Rahmen des Forschungsvorhabens inMEDIASres entwickelt und erprobt wurde. Das Konzept zur Qualifizierung ist - ebenso wie das agile Lernen selbst durch einen iterativen Charakter und erfahrungsorientiertes Lernen gekennzeichnet.

J. Jungclaus $(\bowtie) \cdot$ P. A. Arndt

ZNL TransferZentrum für Neurowissenschaften und Lernen, Ulm, Deutschland

E-Mail: joana.jungclaus@znl-ulm.de; petra.arndt@znl-ulm.de

A. Bauer

ZNL TransferZentrum für Neurowissenschaften und Lernen der Universität Ulm, Ulm, Deutschland

E-Mail: agnesbauer@web.de 


\section{Der methodische Begleiter als Dreh- und Angelpunkt im agilen Lernen}

Der Rolle des methodischen Begleiters kommt im agilen Lernen eine hohe Bedeutung zu. Was die Abläufe und die Methodik des agilen Lernens betrifft, ist er der Experte. Er organisiert Vorbereitung, Durchführung und Nachbereitung eines jeden agilen Lernprojekts und hat in allen diesen Phasen unterschiedlichste Aufgaben und Verantwortungen. Die Praxisumsetzungen des agilen Lernens zeigen, dass eine gute methodische Begleitung besonders ausschlaggebend für einen hohen Outcome und eine hohe Zufriedenheit der Beteiligten ist (siehe Kap. 12). Dazu gehört, die einzelnen Meetings im agilen Lernen (v. a. Planung, Review, Retrospektive) strukturiert zu moderieren, die Instrumente im agilen Lernen zielgerichtet einzusetzen, den Lernprozess der Lernenden im Sinne von „Hilfe zur Selbsthilfe“ zu unterstützen und ggf. zwischen fachlichen Begleitern, Auftraggeber und/oder Lernteam zu vermitteln.

Der methodische Begleiter belässt die Lernenden in ihrer Verantwortung - interveniert aber, wenn grundlegende Prinzipien des agilen Lernens verletzt werden. Das Ausüben dieser Rolle ist auch, wenn nicht sogar vor allem, eine Frage der Haltung. Die Ergebnisse zur Qualitätssicherung im agilen Lernen haben auch gezeigt, dass gute methodische Begleiter über eine ausgeprägte Situationskompetenz verfügen: Sie können flexibel auf unterschiedliche Situationen, Umstände und Rahmenbedingungen eingehen und haben auch ein Gespür dafür, welche Elemente und Instrumente des agilen Lernens sie unter Umständen situationsabhängig adaptieren müssen.

Dass das agile Lernen flexibel und bedarfsbezogen auf verschiedenste Lerninhalte, Lerngegenstände und Zielgruppen angepasst werden kann, erhöht den Anspruch an die Rolle des methodischen Begleiters noch weiter. Er muss aus didaktisch-methodischer Sicht beurteilen können, ob Themen und Zielgruppen sich für das agile Lernen eignen oder eher mit klassischen Lernformen bewältigt werden sollten. Er unterstützt den fachlichen Begleiter bei der Formulierung von Lernaufgaben für ein spezifisches Thema, legt eine sinnvolle Lernorganisation fest u. v. m. Zudem ist es Aufgabe des methodischen Begleiters, die fachlichen Begleiter, den Auftraggeber und die Lernenden auf ihre Rollen vorzubereiten - insbesondere, wenn diese das agile Lernen im Unternehmen bisher nicht kennen.

Es wird deutlich, dass die erfolgreiche Begleitung von Lernteams (und fachlichen Begleitern) im agilen Lernen vielfältige Kompetenzanforderungen an den methodischen Begleiter stellt. Wenn Unternehmen die Rolle des methodischen Begleiters intern besetzen wollen, ist es folglich von zentraler Bedeutung für das Gelingen eines agilen Lernprojekts, diese Person entsprechend auszubilden. Welche Inhalte und Kompetenzen im Einzelnen notwendig sind und wie die Qualifizierung begleitend $\mathrm{zu}$ einem agilen Lernprojekt im Unternehmen erfolgen kann, wird im folgenden Kapitel verdeutlicht.

\subsection{Vorgehen in der Qualifizierung}

Wie im Kap. 11 beschrieben wird, zielt agiles Lernen auf den Aufbau von beruflicher Handlungskompetenz ab - es eignet sich immer dann, wenn Mitarbeitende ihr Wissen in verschiedensten Situationen anwenden, eigenverantwortlich und selbstorganisiert handeln und auf unterschiedliche Randbedingungen reagieren müssen. Überträgt man diese An- 
sprüche auf das Handeln des methodischen Begleiters im agilen Lernen, wird klar, dass dieser ebenfalls Handlungskompetenz zur Begleitung eines agilen Lernprojekts aufbauen muss: Die methodische Begleitung ist wesentlich anspruchsvoller als nur „Kochen nach Rezept“. Aus diesem Grund folgt die Qualifizierung der methodischen Begleiter ähnlichen Leitprinzipien wie das agile Lernen selbst:

Methodische Begleiter lernen erfahrungsorientiert, d. h. sie lernen durch das Tun. Dabei ist wichtig, dass die angehenden Begleiter zunächst nur einzelne Elemente oder Meetings des agilen Lernens verantworten und ihr Handeln reflektieren, um bewusst aus ihren Erfahrungen zu lernen. Dazu wurde im Forschungsprojekt das Verfahren der Prozessbegleitung genutzt: Die methodischen Begleiter wurden durch das Forscherteam qualifiziert und während des agilen Lernprojekts eng durch eine der Forscherinnen begleitet. Diese coachte sie während der ersten Etappen des agilen Lernens, sie begleitete die Reflexion der gemachten Erfahrungen und gab Tipps und Feedback.

Die Qualifizierung der methodischen Begleiter macht sich den iterativen Charakter des agilen Lernens zu nutze. Die Begleiter wachsen nach und nach in die Rolle, indem sie zunehmend mehr Aufgaben in einem agilen Lernprojekt selbst ausführen und verantworten. Dadurch übernehmen sie sukzessive mehr Verantwortung und erwerben nach und nach die vielfältigen Kompetenzen, die für die methodische Begleitung eines agilen Lernprojekts notwendig sind - ohne dabei überfordert zu werden.

Im gesamten Verlauf der Qualifizierung wird die Vorerfahrung eines angehenden methodischen Begleiters berücksichtigt: Jemand, der bereits Erfahrung in der Moderation und Teamentwicklung hat, kann bereits früh eigene Meetings moderieren. Jemand, der Scrum schon aus der Projektorganisation kennt, kann sich schnell in bestimmte Tools des agilen Lernens einfinden (z. B. Kanban-Board).

\subsection{Inhalte und Methoden der Qualifizierung zum methodischen Begleiter}

Das Vorgehen ermöglicht, dass die methodischen Begleiter im Rahmen ihrer Qualifizierung eng begleitet und situationsbezogen gecoacht wurden. Dadurch können sie erfolgreich in ihre Rolle wachsen. Dabei hat es sich bewährt, dass die methodischen Begleiter zuerst für die Durchführungsphase (d. h. die Begleitung der Lernenden) qualifiziert werden, bevor sie Kompetenzen zur Vor- und Nachbereitung eines agilen Lernprojekts erwerben. Darüber hinaus werden bestimmte Inhalte als ein Baustein im Seminarsetting vermittelt - insbesondere Grundlagen zur Haltung eines methodischen Begleiters und zu den Abläufen im agilen Lernen.

\section{Grundlagen zur Rolle und Haltung der methodischen Begleitung}

Der Grundlagenbaustein zum Thema "Rolle und Haltung eines methodischen Begleiters“ umfasst einen halben Tag im Seminarformat. Dieser Baustein wurde durch die Forscherinnen des Forscherteams durchgeführt, die auch später die Rolle der Prozessbegleitung für die angehenden methodischen Begleiter einnahmen. Der Baustein hat folgende Inhalte: 
- Ziele und Lernverständnis im agilen Lernen

- Elemente, Abläufe und Instrumente im agilen Lernen

- Rolle und Aufgaben des methodischen Begleiters

- Haltung eines methodischen Begleiters (inkl. Prinzipien der Lernprozessbegleitung und Grundlagen gelingenden Lernens)

- Techniken, Methoden und Tools zur Kommunikation und Moderation der Meetings im agilen Lernen

- Anwendung der Techniken und Instrumente im Rahmen von Simulationen zum agilen Lernen

In diesem Baustein wird ebenfalls das Prinzip der Erfahrungsorientierung angewandt wenn auch in Form von Rollenspielen und im simulierten Setting. Mit Blick auf die noch folgenden Qualifizierungsteile erwiesen sich die Simulationen als besonders hilfreich, weil die methodischen Begleiter die einzelnen Meetings des agilen Lernens (Planung, Review, Retrospektive) mit ihren Funktionen und Instrumenten auf diese Weise realitätsnah kennenlernen konnten.

Um intensiv an den Inhalten arbeiten zu können, sollten maximal sechs angehende methodische Begleiter an einem Baustein teilnehmen. Die Erfahrung zeigt, dass die Teilnehmer jedoch durchaus unternehmensübergreifend zusammengesetzt sein können. Besonders hilfreich ist, wenn die Teilnehmer vorab Gelegenheit gehabt haben, das agile Lernen kennen zu lernen - z. B. in der Rolle des Lernenden oder wenn sie vor Beginn des Bausteins als Beobachter an einer Etappe eines laufenden agilen Lernprojekts teilnehmen konnten.

\section{Durchführungsphase des agilen Lernens: Ein Lernteam begleiten}

Im nächsten Schritt lernen die methodischen Begleiter, ein Lernteam im agilen Lernen zu begleiten - bei einem Lernthema, für das bereits alle Vorbereitungen getroffen sind. Das heißt, alle Rollenträger sind benannt und in ihre Rolle eingeführt, Lernthema und Lernaufgaben sind bereits umfassend ausgearbeitet und alle technisch-organisatorischen Fragen sind bereits geklärt (z. B. Länge der Etappen).

Der angehende methodische Begleiter nimmt die Funktion zunächst in Personalunion mit seiner Prozessbegleitung ein (coachende Begleitung für den methodischen Begleiter) und übernimmt Etappe für Etappe mehr Aufgaben und Verantwortung. In der ersten Etappe des agilen Lernens moderiert die Prozessbegleitung alle Meetings (Kick-off, Planung, Review, Retrospektive). Der zu qualifizierende Begleiter bekommt vorab Beobachtungsaufträge und reflektiert den Ablauf im Nachgang mit der Prozessbegleitung. Je nach Vorerfahrung kann er in der nächsten Etappe ein oder mehrere Meetings selbst moderieren - nun ist die Prozessbegleitung in der Rolle des Beobachters und kann bei Bedarf während des Meetings unterstützen oder eingreifen. Nach den Meetings reflektieren beide das Handeln des methodischen Begleiters und die Prozessbegleitung gibt Feedback sowie Tipps für die nächste Etappe. Die Inhalte aus dem Grundbaustein werden ebenfalls mit Blick auf ihre Umsetzung im Handeln reflektiert. 
So lernt der angehende methodische Begleiter die einzelnen Meetings strukturiert zu moderieren, die Instrumente des agilen Lernens zur Anwendung zu bringen und seine Haltung zu üben. Im Laufe des Prozesses können einzelne Themen ,nachqualifiziert“ oder spezifische Themen vertieft werden. Die Themen werden am besten im Laufe der Etappen bearbeitet, sodass sie im Rahmen der nächsten Meetings bereits geübt und reflektiert werden können.

Wenn der methodische Begleiter die Haltung gut umsetzt und alle Abläufe und Strukturen des agilen Lernens verinnerlicht hat, können auch erste Schritte in Richtung Situationskompetenz gemacht werden: Durch das Lernen an echten Erfahrungen und Beispielen entstehen auch „echte Situationen“, auf die der methodische Begleiter reagieren muss. Die Prozessbegleitung kann diese gezielt mit dem methodischen Begleiter reflektieren und Handlungsalternativen aufzeigen. Dazu gehört auch, dass Instrumente und Abläufe des agilen Lernens situationsspezifisch durch den methodischen Begleiter angepasst werden können.

Das Konzept zur Qualitätssicherung im agilen Lernen sieht vor, dass der methodische Begleiter darüber hinaus die qualitätsrelevanten Aspekte im agilen Lernen kennt und deren Umsetzung besonders im Blick hat. Dazu zählt u. a., dass er auf ein gutes AufwandNutzen-Verhältnis der einzelnen Meetings im agilen Lernen achtet (siehe Kap. 12). Diese Themen kann ein angehender methodischer Begleiter ebenfalls im Rahmen der Durchführungsphase eines agilen Lernprojekts - erfahrungsorientiert und in Zusammenarbeit mit der Prozessbegleitung - in den weiteren Etappen des agilen Lernens erlernen.

$\mathrm{Ob}$ die Situationskompetenz und die Anforderungen der Qualitätssicherung bereits nach zwei bis drei Etappen vom angehenden methodischen Begleiter beherrscht werden oder ob sie erst nach mehreren agilen Lernprojekten in der Zusammenarbeit zwischen Prozessbegleitung und methodischem Begleiter bearbeitet werden, hängt stark von den Vorkenntnissen und Fähigkeiten des methodischen Begleiters ab. Wenn die Haltung des angehenden methodischen Begleiters bereits zu der Haltung passt, die im agilen Lernen benötigt wird, und der Ablauf schnell verinnerlicht wird, können die Aspekte durchaus im Rahmen eines agilen Lernprojekts, das mehrere Etappen hat, bearbeitet werden.

Die Präsenz der coachenden Begleitung nimmt also nach und nach ab. Ab einem gewissen Zeitpunkt „reichen“ dem methodischen Begleiter kurze Telefonate zur Reflexion, bis er schlussendlich alle benötigten Kompetenzen erworben hat und genügend Erfahrung sammeln konnte, um Lernteams eigenständig zu begleiten.

Die Inhalte und Ziele der Qualifizierung, die begleitend zur Durchführungsphase eines agilen Lernprojekts verfolgt werden, sind auf einen Blick:

- Haltung, Grundlagen gelingenden Lernens und Prinzipien der Lernprozessbegleitung zur Anwendung bringen

- Einüben des Ablaufs und Anwendung der Instrumente im agilen Lernen

- Moderation und Organisation aller Meetings im agilen Lernen und Lernprozessbegleitung der Lernteams

- Situationskompetenz erwerben

- Qualitätsrelevante Aspekte im agilen Lernen kennen und beachten 


\section{Agiles Lernen vor- und nachbereiten}

Die Vor- und Nachbereitung eines agilen Lernprojekts erfordert z. T. andere Kompetenzen als die Durchführungsphase (siehe Kap. 11). Die Qualifizierung zur eigenständigen Durchführung dieser Phasen beginnt, wenn der methodische Begleiter bereits erste agile Lernprojekte in der Durchführungsphase begleitet hat. Durch diese Vorerfahrung fällt es dem angehenden Begleiter leichter nachzuvollziehen, worauf in der Vorbereitungsphase zu achten ist und insbesondere ein Verständnis dafür zu entwickeln, nach welchen Kriterien gute Lernaufgaben entwickelt werden können.

Inhaltlich geht es im Kern darum, das Instruktionsdesign zur Vor- und Nachbereitung von agilem Lernen zu verstehen und für verschiedene Lernthemen umsetzen zu können. Das beinhaltet folgende Aspekte (siehe auch: Kap. 11):

- Kompetenzbedarf klären und auf Eignung für agiles Lernen prüfen

- Zielgruppe, Kultur und Ressourcen klären und auf Eignung prüfen

- Lernthema didaktisch ausarbeiten und Lernaufgaben entwickeln

- Formen didaktischer Lernorganisation ausgestalten und umsetzen

- Rollen ausgestalten und Rollenträger vorbereiten

- Evaluationsziele konkretisieren und Evaluationsinstrumente ausarbeiten

- Im Anschluss an das agile Lernprojekt übergreifende Lessons Learned ableiten und Maßnahmen für folgende agile Lernprojekte planen und umsetzen.

Der methodische Begleiter verantwortet den Gesamtablauf, verteilt mitunter Aufgaben und koordiniert die einzelnen Schritte. So werden die Lernaufgaben beispielsweise von den fachlichen Begleitern formuliert, der methodische Begleiter unterstützt sie jedoch dabei vor dem Hintergrund methodisch-didaktischer Aspekte.

Die Qualifizierung für diese Tätigkeiten sieht zunächst vor, dass der methodische Begleiter die Vor- und Nachbereitung eines Lernthemas als Beobachter begleitet. Alternativ kann die Prozessbegleitung, die den methodischen Begleiter qualifiziert, auf bereits durchgeführte Lernthemen zurückgreifen und dem angehenden methodischen Begleiter die relevanten Unterlagen zur Verfügung stellen (z. B. Lernaufgaben). Anhand dieses Beispiels wird dann vermittelt, welche Aspekte bei der Vor- und Nachbereitung wesentlich sind und worauf zu achten ist.

Darauf aufbauend wird das Leitprinzip der Erfahrungsorientierung und auch die iterative Struktur wieder aufgegriffen. Der methodische Begleiter bereitet ein agiles Lernen vor, das sich aus einem reellen und aktuellen Lernbedarf im Unternehmen ergibt. Die einzelnen Schritte des Instruktionsdesigns sowie die unterstützenden Tools und Leitfäden werden auf dieses Thema angewandt und der methodische Begleiter bereitet dies gemeinsam oder zumindest in regelmäßiger Abstimmung mit seiner Prozessbegleitung vor. Die Prozessbegleitung kann dabei immer wieder Hinweise geben - zum Beispiel zu offenen Klärungsfragen (Lernzielklärung, Rollenbesetzung, Ressourcenplanung etc.) - und auch inhaltlich unterstützen, indem die geplante Lernorganisation, die formulierten Lernauf- 
gaben oder die Evaluationsziele vor Beginn des agilen Lernens mit dem methodischen Begleiter reflektiert werden.

In der Nachbereitung sollte der methodische Begleiter ebenfalls gemeinsam mit seiner Prozessbegleitung reflektieren, welche Teilschritte zufriedenstellend umgesetzt wurden und gut funktionierten und welche Teilschritte für zukünftiges agiles Lernen möglicherweise anders umgesetzt werden sollten. Die gemeinsame Rückschau ermöglicht dem methodischen Begleiter zielgerichtet aus seinen Erfahrungen zu lernen, dadurch Erfahrungswissen aufzubauen und wiederum seine Situationskompetenz zu stärken.

\subsection{Erfahrungen aus der Praxis}

Das Qualifizierungskonzept hat sich im Rahmen des Forschungsvorhabens inMEDIASres bewährt. Insgesamt wurden elf Personen aus den vier Partnerunternehmen nach diesem Vorgehen qualifiziert - allerdings in unterschiedlicher Tiefe. So haben manche der methodischen Begleiter lediglich Kompetenzen und Erfahrungswissen für die Durchführungsphase des agilen Lernens erworben, andere arbeiteten mit ihrer Prozessbegleitung auch zur Vor- und Nachbereitung und können neue Lernthemen eigenständig aufsetzen.

Der Großteil der methodischen Begleiter ist im Personalbereich des Unternehmens beschäftigt und hat bereits Vorerfahrungen in Moderation und der Begleitung von Teams, was sich (ebenso wie eine passende Haltung) als Vorteil für die Qualifizierung erwies.

Durch das iterative Vorgehen und das erfahrungsorientierte Lernen konnten die methodischen Begleiter den Ablauf und die Strukturen des agilen Lernens schnell verinnerlichen. Vor allem mit Blick auf die Haltung haben die methodischen Begleiter stark von der coachenden Begleitung durch ihre Prozessbegleitung profitiert. In den meisten Fällen konnten sie bereits in der zweiten Etappe des agilen Lernens Meetings moderieren und nach wenigen Etappen Aspekte zur Situationskompetenz mit ihrer Prozessbegleitung bearbeiten.

Die Individualität des Vorgehens erwies sich ebenfalls als sehr vorteilhaft. So hat eine angehende methodische Begleiterin im Rahmen ihrer Prozessbegleitung vor allem den Aspekt der Eigenverantwortung bearbeitet (wie belasse ich die Lernenden in ihrer Verantwortung und bin weder überfürsorglich noch überkritisch?) - andere haben sich intensiver mit dem zielgenauen Einsatz verschiedener Instrumente im agilen Lernen befasst, wieder andere hatten wenig Vorkenntnisse in puncto Moderationstechniken und haben diese vertieft.

Durch den hohen Anteil an Reflexion und die direkte Anwendung in der jeweils folgenden Etappe gelang der Aufbau von Handlungskompetenz bei allen methodischen Begleitern gut. Insbesondere der Aufbau von Situationskompetenz im agilen Lernen braucht jedoch Zeit und Erfahrung, weil die Rollenstruktur und die einzelnen Ablaufelemente dazu führen, dass flexibel auf unterschiedlichste Situationen reagiert werden muss. 


\subsection{Fazit und Ausblick}

Jedes agile Lernen braucht einen qualifizierten methodischen Begleiter, an den vielfältige Kompetenzanforderungen gestellt werden. Da neben Techniken und Abläufen vor allem die Haltung und Situationskompetenz entscheidend sind, sollte ein methodischer Begleiter gut qualifiziert sein und insbesondere bei seinem ersten agilen Lernprojekt coachend begleitet werden.

Wenn die unternehmenseigenen methodischen Begleiter alle Qualifizierungselemente durchlaufen haben und bereits Erfahrungswissen aufbauen konnten, können sie auch voneinander lernen und ggf. selbst weitere methodische Begleiter im Unternehmen ausbilden. Im Sinne des Qualitätssicherungskonzepts (siehe Kap. 12) sollte mindestens ein methodischer Begleiter als Process Owner für agiles Lernen im Unternehmen benannt werden. Es empfiehlt sich, dass sich die methodischen Begleiter eines Unternehmens in einem Netzwerk zusammenschließen, um ihr Handeln in diesem Rahmen zu reflektieren und neue Erfahrungswerte zu teilen.

Grundsätzlich ist zu überlegen, inwieweit die methodische Begleitung intern, also aus dem Unternehmen heraus besetzt werden kann und soll. Diese Entscheidung ist neben den dargestellten Kompetenzanforderungen auch von Kapazitätsfragen abhängig - beispielsweise wenn ein Lernbedarf eine oder mehrere Abteilungen betrifft und viele Lernteams in kurzer Zeit lernen müssen.

Da die Durchführungsphase andere Kompetenzen erfordert als die Vor- und Nachbereitung des agilen Lernens, ist bei dieser Entscheidung aber auch eine Zwischenlösung denkbar: Das Unternehmen baut eigene methodische Begleiter für die Durchführungsphase (also zur Begleitung von Lernteams) auf, holt sich für ganz neue Lernthemen jedoch externe Unterstützung zur Vor- und Nachbereitung des agilen Lernens.

Open Access Dieses Kapitel wird unter der Creative Commons Namensnennung - Weitergabe unter gleichen Bedingungen 4.0 International Lizenz (http://creativecommons.org/licenses/ by-sa/4.0/deed.de) veröffentlicht, welche die Nutzung, Vervielfältigung, Bearbeitung, Verbreitung und Wiedergabe in jeglichem Medium und Format erlaubt, sofern Sie den/die ursprünglichen Autor(en) und die Quelle ordnungsgemäß nennen, einen Link zur Creative Commons Lizenz beifügen und angeben, ob Änderungen vorgenommen wurden. Wenn Sie das Buch oder Teile daraus remixen, verändern oder anderweitig direkt darauf aufbauen, dürfen Sie Ihre Beiträge nur unter derselben Lizenz wie das Original verbreiten.

Die in diesem Kapitel enthaltenen Bilder und sonstiges Drittmaterial unterliegen ebenfalls der genannten Creative Commons Lizenz, sofern sich aus der Abbildungslegende nichts anderes ergibt. Sofern das betreffende Material nicht unter der genannten Creative Commons Lizenz steht und die betreffende Handlung nicht nach gesetzlichen Vorschriften erlaubt ist, ist für die oben aufgeführten Weiterverwendungen des Materials die Einwilligung des jeweiligen Rechteinhabers einzuholen.

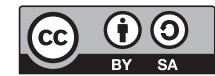

